\title{
Women, innovation, and literature
}

\author{
Desmond D Mascarenhas ${ }^{*}$ and Sally Vander Veer
}

\author{
* Correspondence: \\ desmond@mayflowerworld.org \\ Mayflower Organization for \\ Research and Education, 428 \\ Oakmead Parkway, Sunnyvale, CA \\ 94085, USA
}

\begin{abstract}
Background: Avant-garde literature may influence the evolution of social attitudes and meanings. Women purchase the bulk of literary fiction products in the USA and may thereby play a hitherto undescribed role in business innovation by influencing this type of cultural production. Innovation output in the USA is regionalized and linked to certain psychological traits in local populations. It is reasonable, therefore, to ask whether some of those same psychological traits in women correlate with the consumption of avant-garde literature.

Findings: In a large female cohort, RD scores derived from the eSAIL correlated with geographic regions previously associated with economic innovation. RD values also show significant differences among quartiles of a female cohort scored for innovation based on submissions to an online competition that allowed subjective definitions of 'innovation.' Using online virtual book clubs, RD scores were shown to be significantly closer among pairs of women who had self-selected for similarities in reading taste. Finally, using 724 ratings derived from reader-book pairs, we showed that RD values were significantly different between women who preferred avant-garde literature (11 novels) and those who preferred other long fiction (21 novels), even though average ratings from the reading population at large were nearly identical for both groups of books.

Conclusions: RD values in women correlated with indices of innovation activity and content. These findings may have implications for our understanding of the influence of psychological traits upon the production of cultural attitudes in innovation-dependent economies.

Keywords: eSAlL; Innovation; Avant-garde literature
\end{abstract}

\section{Findings}

Introduction

Innovation occurs in the context of the co-evolution of technology and society. As the primary purchasers of literary fiction in industrialized nations, women may be instrumental in mediating the impact of avant-garde literature upon the production of social meanings and ideas (Jordan-Zachery 2009). However, the differentiated role of women in the evolution of cultural meanings within innovation-based economies has not been described.

On a global level, cultures of innovation may stretch across a field of transnational linkages through which cultural images and meanings are broadcast and have transformative effects. This is demonstrably true, for example, in music and literature (Kong 1995). Inputs to innovation management practice are especially important when

(C) 2014 Mascarenhas and Veer; licensee Springer. This is an Open Access article distributed under the terms of the Creative Commons Attribution License (http://creativecommons.org/licenses/by/2.0), which permits unrestricted use, distribution, and reproduction in any medium, provided the original work is properly credited. 
institutions are viewed not merely as replicative entities providing inertia and stability but as venues for the dynamic interplay between actors and structures informed by cultural meanings (Geels 2004). Corsi and others have argued that innovation management in a globalized economy must take into consideration the cultural capacities of constituents and, in turn, dissemination of knowledge and meanings across cultural boundaries (Corsi 1998). In a microeconomic setting, the impacts of women on innovation may be particularly relevant to the management of family firms. Small family businesses have traditionally been regarded as risk averse, but a recent study showed that innovation explained $44 \%$ of the variance in performance of family firms versus $22 \%$ of the corresponding variance for non-family small- and medium-sized enterprises (Price et al. 2013).

Following the 'linguistic turn' of the twentieth century, the proposition that meaning comes from language has become more or less axiomatic among professional philosophers. The late Richard Rorty famously suggested that meaning in language evolves through avant-garde innovations in literature (Rorty 1989), but a social link between economic innovation and literary innovation has never been formally demonstrated. Indeed, Rorty did not address possible social mechanisms by which psychological traits associated with innovation in general might be instrumental in either the generation or consumption of avant-garde literature in particular, though his theory at least suggests that intriguing possibility.

Could traits associated with economic innovation be instrumental in the generation or consumption of avant-garde literature? If so, and if Rorty's thesis is correct, could the evolution of language and meaning be connected to innovative economic activity via psychological traits? Recent work suggests that business innovation clusters 'work' because they gather cognitive and cultural similarities resulting from shared mental models and business concepts (Pouder and St. John 1996; Gomes and Hurmelinna-Laukkanen 2013). The eSAIL inventory (Mascarenhas et al. 2007), has recently been used to demonstrate significant psychological differences between geographic regions in the USA. Some of this variance can be explained by differences in the occupational mix between regions, but two scales of the inventory, RESPBIAS and DOGMATIC, showed significant regional differences even within members of a single profession (Mascarenhas and Singh 2012). Although a causal relationship was not proven in the study, inhabitants of regions associated with product cycle innovation scored lower, on average, on both scales. In the present study, we reversed and averaged the RESPBIAS and DOGMATIC scales and used this composite scale (RD) to measure a possible relationship with innovation in selected US cohorts of women.

We elected to focus on women for three reasons: (a) the a priori thesis that infants first learn language and cultural meanings from their mothers, (b) women buy approximately two thirds of all literary fiction in the USA (Simba Information 2013), and (c) innovation in small family-owned firms (including women-owned) explains $44 \%$ of the variance in performance of those firms.

\section{Results}

Occupational, cultural, and demographic data were obtained from public online sources, unless otherwise noted. Online data collection from 1,277 anonymous adult participants 
Table 1 RD values of women in different geographic regions

\begin{tabular}{lccc}
\hline Region & Sample $(\boldsymbol{n})$ & Mean RD value & $\boldsymbol{p}$ value \\
\hline 2 & 92 & $-0.403 \pm 0.755$ & \\
3 & 102 & $0.022 \pm 0.658$ & $<0.0001$ \\
6 & 104 & $0.055 \pm 0.789$ & $<0.0002$ \\
\hline
\end{tabular}

Regions 3 and 6 were previously shown to correlate with the generation and assimilation of economic innovation, compared to region 2 (Mascarenhas and Singh 2012). The data shown here are subsets of the original data, with males removed.

(cohorts A to C) was described in a previously published psychometric study that also described the validation of the eSAIL inventory, a 43-item online inventory for which satisfactory Cronbach alpha and test-retest reliability coefficients were observed for all primary psychometric scales, and discriminant, convergent, and predictive validities of scales were shown to be consistent across all cohorts (Mascarenhas et al. 2007). Individuals in these cohorts answered a questionnaire containing demographic questions about age, gender, household income, education, state of residence, profession, and mobility. Those data, as well as eSAIL scores, were used in the present analysis. A subset of the 13 eSAIL scales was designed to measure attributes believed to relate to innovation: plasticity/rigidity (IMPROMPTU, DOGMATIC), internal/external locus of control (BOLD, RESPBIAS), optimism/pessimism (POSITIVE, MACH), and abstraction (ABSTRACT) (Mascarenhas et al. 2007). In the current work, geographic differences in aggregate psychometric scores for regions and occupations were, in all instances, shown to be independent of age, gender, and income. State-wise data were converted to $z$ scores (standard deviations from the national mean). Data are shown as \pm SD. The composite scale RD was derived by averaging the $z$ values for the RESPBIAS and DOGMATIC scales and multiplying the result by -1 (thereby reversing the direction of the resulting scale). Online survey data on reading tastes were collected as previously described (Mascarenhas et al. 2007; Mascarenhas and Singh 2012). The eSAIL is freely available at www.brainterrain.com.

Table 1 shows that regional RD differences previously shown for the whole population are also observed when women are separately analyzed. Regions 3 and 6, associated with economic innovation, score significantly higher than region 2 , as shown in the original study for the whole population (Mascarenhas and Singh 2012). However, does RD correlate with self-defined personal measures of innovation in women? Table 2 shows that when women are stratified into quartiles based on an innovation contest judged by a panel of women, their RD scores correlate strongly with innovation scores.

Table 2 RD values and personal innovation

\begin{tabular}{lcc}
\hline Quartile & Innovation score & Mean RD value \\
\hline Q1 $(n=29)$ & $-0.733 \pm 0.181$ & $0.085 \pm 0.817$ \\
Q2 $(n=29)$ & $-0.298 \pm 0.117$ & $0.346 \pm 0.618$ \\
Q3 $(n=29)$ & $0.030 \pm 0.146$ & $0.277 \pm 0.723$ \\
Q4 $(n=29)$ & $1.017 \pm 0.619^{* *}$ & $0.550 \pm 0.527^{*}$ \\
\hline
\end{tabular}

${ }^{*} p<0.02$ vs. Q1; ${ }^{* *} p<0.01$ vs. Q1. Self reports of individually initiated innovation projects by a cohort of 116 women were scored for innovation merit by a panel of female professionals from five disciplines (anthropology, scientific research (evolutionary biology), research technician, marketing executive, education professional). The cohort was divided into quartiles based on innovation $z$ score. 
Table 3 RD values and reading preferences

\begin{tabular}{|c|c|c|c|}
\hline Type of pair & Sample $(n)$ & Mean RD value & $p$ value \\
\hline Matched buddy & 95 & $0.665 \pm 0.476$ & \\
\hline Random & 95 & $0.894 \pm 0.719$ & 0.010 \\
\hline
\end{tabular}

If reading tastes are related to $\mathrm{RD}$ at all, we should observe more similar $\mathrm{RD}$ values for readers with matched tastes than for shuffled (random) pairs. An online cohort of women used a 'virtual book club' website and posted ratings and reviews of novels they had read over a period of several weeks. Based on these reviews, each woman selected a 'matched buddy' with similar reading tastes. We hypothesized that matched pairs should exhibit average differences in RD score that are significantly smaller than the RD difference between randomly matched pairs generated by shuffling the same individuals. Table 3 shows data in support of this prediction.

We arbitrarily defined 11 novels that were winners of the Pulitzer Prize and National Book Award as 'avant-garde' literature. The control group, on the other hand, consisted of 25 novels that received equally high ratings from readers but did not win major awards. Table 4 shows that women readers who give avant-garde literary fiction a maximum rating have significantly higher RD values than women who favor other types of equally 'good' fiction, even though the average rating given by all readers for the books in each category is essentially identical (mean $4.49 \pm 0.13$ vs. $4.45 \pm 0.18$; difference not statistically significant).

\section{Conclusions}

Taken together, the above data provide a possible mechanism for Rorty's core proposition regarding innovation in language, at least insofar as women are concerned. It is important to remember that our data are only correlational. Although RD seems to correlate with innovation activity, and we can measure it, we do not know what underlying psychological processes it might represent. Moreover, much further study will be needed to establish whether, in fact, there is any causal relationship between RD and the evolution of language. Nevertheless, measures such as RD may serve as simple and useful diagnostics for exploration within this area of inquiry.

The practical implications of this finding, if confirmed by future studies, are potentially significant for innovation management. They suggest the importance of adaptive psychological traits in the 'innovation component' of economic performance. Using the NEO-FF personality inventory, the association of psychological traits with innovation has been suggested by other investigators (Steel et al. 2012), but our study uses

Table 4 RD values and appreciation of avant-garde literature

\begin{tabular}{lccr}
\hline Category & Ratings $(\boldsymbol{n})$ & Mean RD value & $\boldsymbol{p}$ value \\
\hline Avant-garde & 204 & $0.878 \pm 0.177$ & \\
Other & 564 & $0.705 \pm 0.163$ & 0.0022 \\
\hline
\end{tabular}

Eleven novels that received a Pulitzer Prize or National Book Award were compared with 21 novels that did not receive major awards but were rated by readers, on average, just as highly (mean $4.49 \pm 0.13$ vs. $4.45 \pm 0.18$ ). The mean RD value is shown for readers who gave books in each category a rating of 5 , the highest rating. 
the eSAIL inventory, which was designed specifically for the measurement of adaptive traits (Mascarenhas et al. 2007). Women often play a key role in small family-owned businesses. A recent study showed that innovation explained $44 \%$ of the performance variance of small family firms (Price et al. 2013). Beyond that, in a larger cultural sense, women with adaptive traits may play a key role in bringing new cultural attitudes and meanings into innovation-dependent economies, both because of their role in teaching language to infants and because of their financial support of avant-garde literature.

Competing interests

The authors declare that they have no competing interests.

Authors' contributions

DM designed the investigations, performed data collection and analysis, implemented software design, and wrote the manuscript. SV helped manage online database content. All authors read and approved the final manuscript.

Received: 16 September 2013 Accepted: 4 February 2014

Published: 21 Feb 2014

\section{References}

Corsi, C. (1998). A global science and technology world: a strategic tool for market globalization. In C Corsi \& SV Kudrya (Eds.), Globalization of science and technology: a way for C.I.S. countries to new markets (pp. 15-26). Dordrecht: Kluwer.

Geels, FW. (2004). From sectoral systems of innovation to socio-technical systems: Insights about dynamics and change from sociology and institutional theory. Research Policy, 33, 897-920.

Gomes, J, \& Hurmelinna-Laukkanen, P. (2013). Behind innovation clusters: individual, cultural and strategic linkages. International Journal of Innovation Science, 5(2), 89-102.

Information, S. (2013). Business of consumer book publishing 2013. Rockville: Simba Information.

Jordan-Zachery, JS. (2009). Perceptions, culture and policy: a racing-gendering perspective. In JS Jordan-Zachery (Ed.), Black women, cultural images and social policy (pp. 9-11). New York: Taylor \& Francis.

Kong, L. (1995). Popular music in geographical analyses. Progress in Human Geography, 19, 183-198.

Mascarenhas, D, \& Singh, AH. (2012). Regional culture and adaptive behavior of physicians. Journal of Bioeconomics, 14(3), 257-266.

Mascarenhas, D, Singh, BK, Singh, AH, \& Veer, SV. (2007). Early adoption of new drug treatments: the role of continuing medical education and physician adaptivity. Critical Pathways Cardiology, 6(1), 30-40.

Pouder, R, \& St. John, CH. (1996). Hot spots and blind spots: geographical clusters of firms and innovation. The Academy of Management Review, 21(4), 1192-1225.

Price, DP, Stoica, M, \& Boncella, RJ. (2013). The relationship between innovation, knowledge, and performance in family and non-family firms: an analysis of SMEs. Journal of Innovation and Entrepreneurship, 2, 14.

Rorty, R. (1989). Contingency, irony, and solidarity. Cambridge: Cambridge University Press.

Steel, G, Rinne, T, \& Fairweather, J. (2012). Personality, nations, and innovation: Relationships between personality traits and national innovation scores. Cross-Cultural Research, 46, 3.

10.1186/2192-5372-3-7

Cite this article as: Mascarenhas and Veer: Women, innovation, and literature. Journal of Innovation and

Entrepreneurship 2014, 3:7

\section{Submit your manuscript to a SpringerOpen ${ }^{\circ}$ journal and benefit from:}

- Convenient online submission

- Rigorous peer review

- Immediate publication on acceptance

- Open access: articles freely available online

- High visibility within the field

- Retaining the copyright to your article

Submit your next manuscript at $>$ springeropen.com 